\title{
Resonant Sturm-Liouville Boundary Value Problems for Differential Systems in the Plane
}

\author{
Alberto Boscaggin and Maurizio Garrione
}

\begin{abstract}
We study the Sturm-Liouville boundary value problem associated with the planar differential system $J z^{\prime}=\nabla V(z)+R(t, z)$, where $V(z)$ is positive and positively 2-homogeneous and $R(t, z)$ is bounded. Assuming Landesman-Lazer type conditions, we obtain the existence of a solution in the resonant case. The proofs are performed via a shooting argument. Some applications to boundary value problems associated with scalar second order asymmetric equations are discussed.
\end{abstract}

Keywords. Positively homogeneous systems, Sturm-Liouville boundary value problems, resonance, Landesman-Lazer conditions

Mathematics Subject Classification (2010). Primary 34B15, secondary 37E45, $37 \mathrm{~J} 45$

\section{Introduction}

The study of boundary value problems at resonance is a very classical topic in the theory of nonlinear differential equations. The pioneering work in this field carries the names of Landesman and Lazer [16], who provided, in 1970, an existence result for the Dirichlet problem associated with the elliptic PDE

$$
\Delta u+\lambda u+r(x, u)=0, \quad x \in \Omega \subset \mathbb{R}^{N},
$$

where $\lambda$ is an eigenvalue of $-\Delta$ on $\Omega$ and $r(x, u)$ is a bounded function. More precisely, the function $r(x, u)$ was there required to satisfy an integral assumption which is nowadays known as the Landesman-Lazer condition. From there on, results of Landesman-Lazer type have been given by many authors, both

A. Boscaggin: Dipartimento di Matematica, Università di Torino, 10123 Torino, Italy; alberto.boscaggin@unito.it

M. Garrione: Dipartimento di Matematica ed Applicazioni, Università di Milano Bicocca, 20125 Milano, Italy; maurizio.garrione@unimib.it 
for ordinary and partial differential operators, and with different boundary conditions (see, for instance, the bibliography in [10]).

In particular, after the works by Dancer [4] and Fučik [13], a great deal of interest was devoted to resonance with respect to a more general kind of spectrum, replacing the notion of eigenvalue $\lambda \in \mathbb{R}$ with pairs $(\mu, \nu) \in \mathbb{R}^{2}$ such that the homogeneous asymmetric equation

$$
\Delta u+\mu u^{+}-\nu u^{-}=0,
$$

with $u^{ \pm}=\max \{ \pm u, 0\}$, has a nontrivial solution satisfying the prescribed boundary conditions. The set of all such couples $(\mu, \nu)$ is known as the DancerFučik spectrum. As for the solvability of nonlinear perturbations of (1) under conditions of Landesman-Lazer type, focusing especially on the case of ordinary differential operators, we mention the papers $[1,5-7,17]$ (see also the bibliography in [15]).

In this paper, we deal with resonant Sturm-Liouville type boundary value problems for first order planar differential systems like

$$
J z^{\prime}=\nabla V(z)+R(t, z), \quad t \in[0, T], z \in \mathbb{R}^{2},
$$

where $J=\left(\begin{array}{cc}0 & -1 \\ 1 & 0\end{array}\right)$ is the standard symplectic matrix, $R:[0, T] \times \mathbb{R}^{2} \rightarrow \mathbb{R}^{2}$ is bounded, and $V$ is a positive and positively 2-homogeneous function (see formula (5) below). As first noticed in [9], this framework provides an elegant extension of the scalar second order ODE

$$
u^{\prime \prime}+\mu u^{+}-\nu u^{-}+r(t, u)=0,
$$

since an analogous of the concept of resonance for $u^{\prime \prime}+\mu u^{+}-\nu u^{-}=0$ can be introduced for the autonomous Hamiltonian problem $J z^{\prime}=\nabla V(z)$. This issue was extensively studied with respect to $T$-periodic boundary conditions, and several existence results (both under nonresonance and resonance assumptions) for (2) were given, e.g., in [8-10,12]. More recently, the Sturm-Liouville boundary value problem for (2) was considered; precisely, in [11], the boundary conditions

$$
z(0) \in l_{S}, \quad z(T) \in l_{A},
$$

where $l_{S}$ and $l_{A}$ are two lines passing through the origin, were taken into account, and the existence of a solution to (2)-(4) was provided in a nonresonant setting.

The aim of the present work is to give, on the other hand, a LandesmanLazer type result in the resonant case. The corresponding statement in the periodic framework was obtained in [10], using a degree theoretical approach. Here, instead, we take advantage of a sharp dynamical interpretation of the Landesman-Lazer condition first developed in [3] (see Remark 2.7), and we tackle our problem by means of an elementary planar shooting technique. This is done in detail in Section 2, while in Section 3 we give some corollaries dealing with Sturm-Liouville boundary value problems associated with (3), to be compared with the results in $[1,5,6]$. 


\section{The main result}

2.1. Preliminaries. Let us start by recalling the setting used in $[9,11]$. We denote by $\mathcal{P}$ the set of the $C^{1}$-functions $V: \mathbb{R}^{2} \rightarrow \mathbb{R}$, with locally Lipschitz continuous gradient, which are positively homogeneous of degree 2 and positive, i.e.,

$$
0<V(\lambda z)=\lambda^{2} V(z), \quad \lambda>0, z \in \mathbb{R}^{2} \backslash\{0\} .
$$

Fixed $V \in \mathcal{P}$, the solutions to

$$
J z^{\prime}=\nabla V(z)
$$

describe strictly star-shaped Jordan curves around the origin, covered in clockwise sense. Moreover, as a consequence of the 2-homogeneity, fixed $\theta_{1}, \theta_{2}$ in $[0,2 \pi]$ with $\theta_{1} \leq \theta_{2}$, the time needed for the solutions to cover exactly the angular sector $\theta_{1} \leq \theta \leq \theta_{2}$ is independent of the norm of the starting point and is given by

$$
\int_{\theta_{1}}^{\theta_{2}} \frac{d \theta}{2 V(\cos \theta, \sin \theta)}
$$

In particular, the origin is an isochronous center for (6), i.e., there exists $\tau_{V}>0$ such that all the nontrivial solutions to (6) have minimal period equal to $\tau_{V}$. Finally, notice that once a nontrivial solution $\varphi_{V}(t)$ to $(6)$ is chosen, all the other ones are of the form $u(t)=C \varphi_{V}(t+\theta)$, where $C \geq 0, \theta \in\left[0, \tau_{V}\left[\right.\right.$. We can fix $\varphi_{V}$ such that $\varphi_{V}(0)$ belongs to the vertical positive semi-axis and $V\left(\varphi_{V}(t)\right) \equiv \frac{1}{2}$.

We now turn our attention to the autonomous Sturm-Liouville problem

$$
\left\{\begin{array}{c}
J z^{\prime}=\nabla V(z) \\
z(0) \in l_{S}, z(T) \in l_{A},
\end{array}\right.
$$

where $l_{S}$ and $l_{A}$ are two lines passing through the origin in the plane ("S" stands for "starting" and "A" for "arrival"). We will say that (8) is resonant if it has at least a nontrivial solution; since $V \in \mathcal{P}$, it is possible to well characterize this concept, as we are going to see. To this end, let us focus on $\varphi_{V}(t)$, for $t \geq 0$. We denote by $\tau_{0, V}$ the least nonnegative time instant such that $\varphi_{V}\left(\tau_{0, V}\right) \in l_{S}$ and we set:

- $\tau_{1, V}>0$ as the least positive time such that $\varphi_{V}\left(\tau_{0, V}+\tau_{1, V}\right) \in l_{A}$;

- $\sigma_{1, V} \geq 0$ as the least nonnegative time such that $\varphi_{V}\left(\tau_{0, V}+\tau_{1, V}+\sigma_{1, V}\right) \in l_{S}$;

- $\tau_{2, V}>0$ as the least positive time such that $\varphi_{V}\left(\tau_{0, V}+\tau_{1, V}+\sigma_{1, V}+\tau_{2, V}\right) \in l_{A}$;

- $\sigma_{2, V} \geq 0$ as the least nonnegative time such that $\varphi_{V}\left(\tau_{0, V}+\tau_{1, V}+\sigma_{1, V}+\tau_{2, V}+\sigma_{2, V}\right) \in l_{S}$. 
In this way, a complete revolution around the origin is performed in the time

$$
\tau_{V}=\tau_{1, V}+\sigma_{1, V}+\tau_{2, V}+\sigma_{2, V}
$$

Notice moreover that, if the lines $l_{S}$ and $l_{A}$ coincide, then it holds that $\sigma_{1, V}=$ $\sigma_{2, V}=0$. We illustrate such definitions in Figure 1 below.

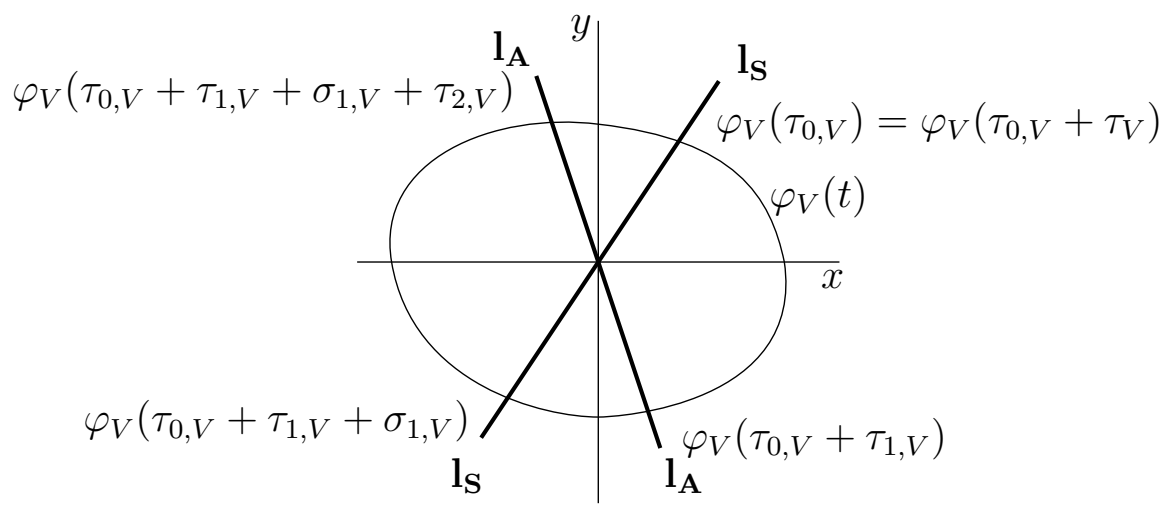

Figure 1: The definition of $\tau_{1, V}, \sigma_{1, V}, \tau_{2, V}, \sigma_{2, V}$.

It is thus easy to see that problem (8) is resonant if and only if, for some nonnegative integer $k$, one of the following four situations occurs:

$$
\begin{aligned}
k \tau_{V}+\tau_{1, V} & =T \\
k \tau_{V}+\tau_{1, V}+\sigma_{1, V}+\tau_{2, V} & =T \\
k \tau_{V}+\tau_{2, V} & =T \\
k \tau_{V}+\tau_{2, V}+\sigma_{2, V}+\tau_{1, V} & =T .
\end{aligned}
$$

Precisely, an eigenfunction (namely, a nontrivial solution to (8)) corresponding to (9) and (10) is always given by

$$
\Phi(t)=\varphi_{V}\left(t+\tau_{0, V}\right),
$$

while an eigenfunction corresponding to (11) and (12) is always given by

$$
\Psi(t)=\varphi_{V}\left(t+\tau_{0, V}+\tau_{1, V}+\sigma_{1, V}\right) .
$$

Notice that, when two or more among the values $\tau_{i, V}, \sigma_{i, V}$ coincide - possibly implying that two or more among the conditions (9)-(12) coincide, as well $\Phi(t)$ and $\Psi(t)$ can be both eigenfunctions at the same time.

2.2. Statement of the main result. We now turn to the nonlinear SturmLiouville boundary value problem

$$
\left\{\begin{array}{c}
J z^{\prime}=\nabla V(z)+R(t, z) \\
z(0) \in l_{S}, z(T) \in l_{A},
\end{array}\right.
$$


where $R:[0, T] \times \mathbb{R}^{2} \rightarrow \mathbb{R}^{2}$ is a Carathéodory function (i.e., measurable in $t$ for every $z$ and continuous in $z$ for almost every $t$ ) which is $L^{1}$-bounded in the $z$-variable, that is, for some $\eta \in L^{1}(0, T)$,

$$
|R(t, z)| \leq \eta(t)
$$

for almost every $t \in[0, T]$ and every $z \in \mathbb{R}^{2}$.

We introduce the following notation: for every $\theta \in \mathbb{R}$,

$$
\begin{aligned}
& \mathcal{J}^{-}(\theta)=\int_{0}^{T} \liminf _{(\lambda, \omega) \rightarrow(+\infty, \theta)}\left\langle R\left(t, \lambda \varphi_{V}(t+\omega)\right) \mid \varphi_{V}(t+\omega)\right\rangle d t, \\
& \mathcal{J}^{+}(\theta)=\int_{0}^{T} \limsup _{(\lambda, \omega) \rightarrow(+\infty, \theta)}\left\langle R\left(t, \lambda \varphi_{V}(t+\omega)\right) \mid \varphi_{V}(t+\omega)\right\rangle d t .
\end{aligned}
$$

Notice that the functions $\mathcal{J}^{ \pm}$are $\tau_{V}$-periodic and satisfy $\mathcal{J}^{-}(\theta) \leq \mathcal{J}^{+}(\theta)$ for every $\theta$. With this position, we now state our main result.

Theorem 2.1. Let $V \in \mathcal{P}$ satisfy (9), for some nonnegative integer $k$, and $R(t, z)$ satisfy (16). Then, the solvability of problem (15) is ensured in each of the following situations:

$$
\begin{array}{lll}
\tau_{1, V}<\tau_{2, V} & \text { and } \quad & \mathcal{J}^{+}\left(\tau_{0, V}\right)<0 \\
\tau_{1, V}>\tau_{2, V} & \text { and } \quad & \mathcal{J}^{-}\left(\tau_{0, V}\right)>0 ; \\
\tau_{1, V}=\tau_{2, V} & \text { and } \quad & \mathcal{J}^{-}\left(\tau_{0, V}\right)>0, \mathcal{J}^{-}\left(\tau_{0, V}+\tau_{1, V}+\sigma_{1, V}\right)>0 \\
\tau_{1, V}=\tau_{2, V} & \text { and } \quad & \mathcal{J}^{+}\left(\tau_{0, V}\right)<0, \mathcal{J}^{+}\left(\tau_{0, V}+\tau_{1, V}+\sigma_{1, V}\right)<0 .
\end{array}
$$

Remark 2.2. The assumptions on $\mathcal{J}^{ \pm}(\theta)$ in (17), (18), (19) and (20) are Landesman-Lazer type conditions, inspired by their counterparts introduced in [10] for the $T$-periodic problem. However, while in [10] the solvability condition reads as

$$
\mathcal{J}^{+}(\theta)<0 \text { for every } \theta \in \mathbb{R} \quad \text { or } \quad \mathcal{J}^{-}(\theta)>0 \text { for every } \theta \in \mathbb{R},
$$

here only special values of $\theta$ must be considered, namely $\theta_{1}=\tau_{0, V}$ and $\theta_{2}=\tau_{0, V}+\tau_{1, V}+\sigma_{1, V}$. This is not surprising, since the Landesman-Lazer conditions usually involve the eigenfunctions of the unperturbed problem, and the nontrivial solutions to (8) are necessarily multiples of $\varphi\left(t+\theta_{i}\right)$, for at least one (possibly both) index $i \in\{1,2\}$ (compare with (13) and (14); of course, in the $T$-periodic case all the functions of the form $\varphi(t+\theta)$ are eigenfunctions).

On the other hand, while in the periodic case the conditions $\mathcal{J}^{+}<0$ and $\mathcal{J}^{-}>0$ are always both admissible, here a precise one is needed according to the statement above. This is not a matter of technicality, on the contrary it is the main feature of Sturm-Liouville boundary value problems, admitting in principle wider resonance regions than the $T$-periodic one (see the discussion in Remark 3.4). 
Similar results when (10), (11) or (12) hold (instead of (9)) can be stated. The precise conditions to be assumed derive clearly from the arguments in the proof below, but maybe they are not obvious at first sight. For this reason, we include all the explicit statements in the theorem below.

Theorem 2.3. Let $V \in \mathcal{P}$ and $R(t, z)$ satisfy (16). Then, the solvability of problem (15) is ensured in each of the following situations:

1) if $V$ satisfies (10) for some nonnegative integer $k$,

$$
\begin{array}{lll}
\sigma_{1, V}<\sigma_{2, V} & \text { and } \quad & \mathcal{J}^{+}\left(\tau_{0, V}\right)<0 \\
\sigma_{1, V}>\sigma_{2, V} & \text { and } \quad & \mathcal{J}^{-}\left(\tau_{0, V}\right)>0 \\
\sigma_{1, V}=\sigma_{2, V} & \text { and } \quad & \mathcal{J}^{-}\left(\tau_{0, V}\right)>0, \mathcal{J}^{-}\left(\tau_{0, V}+\tau_{1, V}+\sigma_{1, V}\right)>0 \\
\sigma_{1, V}=\sigma_{2, V} & \text { and } \quad & \mathcal{J}^{+}\left(\tau_{0, V}\right)<0, \mathcal{J}^{+}\left(\tau_{0, V}+\tau_{1, V}+\sigma_{1, V}\right)<0
\end{array}
$$

2) if $V$ satisfies (11) for some nonnegative integer $k$,

$$
\begin{array}{lll}
\tau_{1, V}>\tau_{2, V} & \text { and } & \mathcal{J}^{+}\left(\tau_{0, V}+\tau_{1, V}+\sigma_{1, V}\right)<0 \\
\tau_{1, V}<\tau_{2, V} & \text { and } \quad \mathcal{J}^{-}\left(\tau_{0, V}+\tau_{1, V}+\sigma_{1, V}\right)>0 ; \\
\tau_{1, V}=\tau_{2, V} & \text { and } \quad \mathcal{J}^{-}\left(\tau_{0, V}\right)>0, \mathcal{J}^{-}\left(\tau_{0, V}+\tau_{1, V}+\sigma_{1, V}\right)>0 \\
\tau_{1, V}=\tau_{2, V} & \text { and } \quad \mathcal{J}^{+}\left(\tau_{0, V}\right)<0, \mathcal{J}^{+}\left(\tau_{0, V}+\tau_{1, V}+\sigma_{1, V}\right)<0
\end{array}
$$

3) if $V$ satisfies (12) for some nonnegative integer $k$,

$$
\begin{array}{lll}
\sigma_{1, V}>\sigma_{2, V} & \text { and } & \mathcal{J}^{+}\left(\tau_{0, V}+\tau_{1, V}+\sigma_{1, V}\right)<0 \\
\sigma_{1, V}<\sigma_{2, V} & \text { and } \quad & \mathcal{J}^{-}\left(\tau_{0, V}+\tau_{1, V}+\sigma_{1, V}\right)>0 \\
\sigma_{1, V}=\sigma_{2, V} & \text { and } \quad & \mathcal{J}^{-}\left(\tau_{0, V}\right)>0, \mathcal{J}^{-}\left(\tau_{0, V}+\tau_{1, V}+\sigma_{1, V}\right)>0 \\
\sigma_{1, V}=\sigma_{2, V} & \text { and } \quad & \mathcal{J}^{+}\left(\tau_{0, V}\right)<0, \mathcal{J}^{+}\left(\tau_{0, V}+\tau_{1, V}+\sigma_{1, V}\right)<0
\end{array}
$$

2.3. Proof of the main result. We argue in an intuitive and informal way, looking at Figure 2 below. As a notation, we denote by $l_{S}^{i}$ and $l_{A}^{i}$, respectively $(i=1,2)$, the two half-lines of $l_{S}$ and $l_{A}$, and by $C^{+}, C^{-}$the two connected components of $\mathbb{R}^{2} \backslash l_{A}$.

First, we make a brief digression about the behavior of the solutions to $J z^{\prime}=\nabla V(z)$ starting on $l_{S}$, following their evolution through the flow from $t=0$ to $t=T\left(=k \tau_{V}+\tau_{1, V}\right)$. In particular, any solution $z_{1}(t)$ starting on $l_{S}^{1}$ performs $k$ clockwise turns around the origin and then covers the angular sector between $l_{S}^{1}$ and $l_{A}^{1}$, thus arriving exactly on $l_{A}^{1}$. On the other hand, any solution $z_{2}(t)$ starting on $l_{S}^{2}$ makes exactly $k$ clockwise turns around the origin in the time $k \tau_{V}$ and then proceeds clockwise for a time equal to $\tau_{1, V}$. The mutual position between $z_{2}(T)$ and $l_{A}^{2}$ is thus determined by the comparison between $\tau_{1, V}$ and $\tau_{2, V}$. Precisely, we have the following possibilities: 


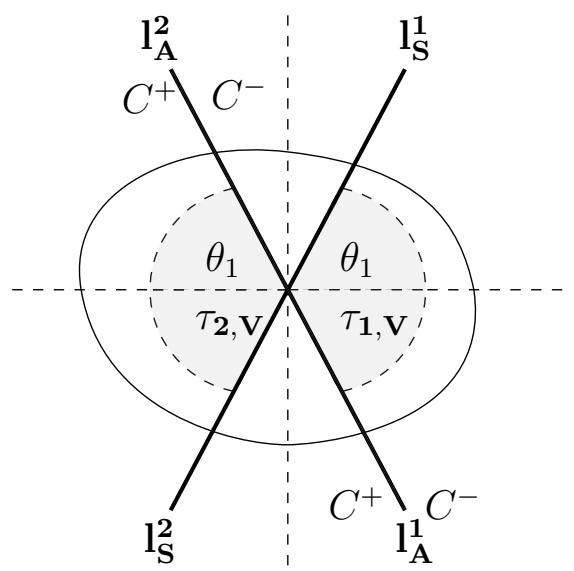

Figure 2: An illustration of the notation used along the proof of Theorem 2.1.

i) if $\tau_{1, V}<\tau_{2, V}, z_{2}(T)$ does not reach $l_{A}^{2}$, so that $z_{2}(t) \in C^{+}$;

ii) if $\tau_{1, V}>\tau_{2, V}, z_{2}(T)$ exceeds $l_{A}^{2}$, but it cannot reach $l_{A}^{1}$, i.e., $z_{2}(T) \in C^{-}$;

iii) if $\tau_{1, V}=\tau_{2, V}$, then $z_{2}(T)$ lies on $l_{A}^{2}$.

We now turn to the nonlinear system

$$
J z^{\prime}=\nabla V(z)+R(t, z)
$$

Having in mind a shooting argument, the existence of a solution to (15) is guaranteed if "large" solutions to $(21)$ starting (for $t=0)$ on $l_{S}^{1}$ and $l_{S}^{2}$ arrive, after the time $T$, into different connected components of $\mathbb{R}^{2} \backslash l_{A}$. To check when this is the case, we always use the fact that the angular component of large solutions to (21) is near the one of the solutions to the unperturbed problem (since $R(t, z)$ satisfies (16)). Hence, on one hand the solutions starting on $l_{S}^{1}$ are "dangerously" near $l_{A}^{1}$. On the other hand, with reference to the previous cases, we can infer that

i) the solutions starting on $l_{S}^{2}$ do not reach $l_{A}^{2}$, thus lying in $C^{+}$. Hence, assuming that $\mathcal{J}^{+}\left(\tau_{0, V}\right)<0$, we will "brake" the solutions starting on $l_{S}^{1}$ so as to make them lie in $C^{-}$;

ii) the solutions starting on $l_{S}^{2}$ exceed $l_{A}^{2}$, thus lying in $C^{-}$. Hence, assuming that $\mathcal{J}^{-}\left(\tau_{0, V}\right)>0$, we will "push" the solutions starting on $l_{S}^{1}$ towards $C^{+}$;

iii) both the solutions starting on $l_{S}^{1}$ and $l_{S}^{2}$, respectively, are dangerously near $l_{A}^{1}$ and $l_{A}^{2}$, respectively, so that we either "brake" them both (assuming $\left.\mathcal{J}^{+}\left(\tau_{0, V}\right)<0, \mathcal{J}^{+}\left(\tau_{0, V}+\tau_{1, V}+\sigma_{1, V}\right)<0\right)$ or "push" them both (assuming $\left.\mathcal{J}^{-}\left(\tau_{0, V}\right)>0, \mathcal{J}^{-}\left(\tau_{0, V}+\tau_{1, V}+\sigma_{1, V}\right)>0\right)$.

All the details of this shooting argument (including the approximation of $R(t, z)$ with Lipschitz functions, so as to have uniqueness for the Cauchy problems) can be found in [11, Theorem 2.1]. It remains to prove that the Landesman-Lazer 
conditions introduced above actually have the desired braking and pushing effects, as shown in the following lemma.

Lemma 2.4. Assume (9) and (16). Then, there exists $R>0$ such that all the solutions $z_{1}(t)$ to $(21)$ with $z_{1}(0) \in l_{S}^{1},\left|z_{1}(0)\right|>R$, satisfy the following:

$$
\begin{array}{lll}
\mathcal{J}^{-}\left(\tau_{0, V}\right)>0 & \Rightarrow & z_{1}(T) \in C^{+} \\
\mathcal{J}^{+}\left(\tau_{0, V}\right)<0 & \Rightarrow & z_{1}(T) \in C^{-}
\end{array}
$$

If, moreover, $\tau_{1, V}=\tau_{2, V}$, then all the solutions $z_{2}(t)$ to $(21)$ with $z_{2}(0) \in l_{S}^{2}$, $\left|z_{2}(0)\right|>R$, satisfy the following:

$$
\begin{array}{lll}
\mathcal{J}^{-}\left(\tau_{0, V}+\tau_{1, V}+\sigma_{1, V}\right)>0 & \Rightarrow & z_{2}(T) \in C^{-} \\
\mathcal{J}^{+}\left(\tau_{0, V}+\tau_{1, V}+\sigma_{1, V}\right)<0 & \Rightarrow & z_{2}(T) \in C^{+}
\end{array}
$$

Proof. We only prove the first claim, the others being analogous. By contradiction, assume that there exists a sequence of solutions $z_{1, n}(t)=z_{n}(t)$, with $\left|z_{n}(0)\right| \rightarrow+\infty$ for $n \rightarrow+\infty$, such that $z_{n}(T) \in C^{-} \cup l_{A}$. By Gronwall's lemma, $\min _{t \in[0, T]}\left|z_{n}(t)\right| \rightarrow+\infty$, so that we can pass to polar coordinates, writing $z_{n}(t)=\rho_{n}(t)\left(\cos \theta_{n}(t), \sin \theta_{n}(t)\right)$. We are going to show that there exists $\bar{n} \in \mathbb{N}$ such that, for every $n \geq \bar{n}, \theta_{n}(T)$ satisfies

$$
\int_{\theta_{n}(0)}^{\theta_{n}(T)} \frac{d \theta}{2 V(\cos \theta, \sin \theta)}>T=k \tau_{V}+\tau_{1, V}
$$

This readily implies the thesis. Indeed, the left-hand side in (22) is the time spent by the solutions to $J z^{\prime}=\nabla V(z)$ to cover the angular sector between $\theta_{n}(0)$ and $\theta_{n}(T)$ (cf. (7)); hence, looking again at Figure 2, (22) means that $z_{n}(t)$ has covered, in the time $T$, a clockwise angular width greater than $2 k \pi+\theta_{1}$. We thus have $z_{n}(T) \in C^{+}$(the fact that $z_{n}(t)$ cannot overcome $l_{A}^{2}$ comes from (16)).

To prove (22), we first compute the angular speed of $z_{n}(t)$. Exploiting the positive 2-homogeneity of $V$, we have

$$
-\theta_{n}^{\prime}(t)=\frac{\left\langle J z_{n}^{\prime}(t) \mid z_{n}(t)\right\rangle}{\left|z_{n}(t)\right|^{2}}=2 V\left(\cos \theta_{n}(t), \sin \theta_{n}(t)\right)+\frac{\left\langle R\left(t, z_{n}(t)\right) \mid z_{n}(t)\right\rangle}{\left|z_{n}(t)\right|^{2}},
$$

so that

$$
\int_{\theta_{n}(0)}^{\theta_{n}(T)} \frac{d \theta}{2 V(\cos \theta, \sin \theta)}=T+\int_{0}^{T} \frac{\left\langle R\left(t, z_{n}(t)\right) \mid z_{n}(t)\right\rangle}{2 V\left(z_{n}(t)\right)} d t=: T+\mathcal{R}_{n} .
$$

It is thus sufficient to show that the second summand in the right-hand side is strictly positive for every $n$ large.

Assume by contradiction that this is not true, namely $\mathcal{R}_{n} \leq 0$ for $n$ large. 
Setting $w_{n}=\frac{z_{n}}{\left\|z_{n}\right\|_{\infty}}$, a standard compactness argument based on the DunfordPettis theorem gives the existence of $w \in C^{1}([0, T])$, with $\|w\|_{\infty}=1$, such that $w_{n} \rightarrow w$ uniformly and $w$ solves $J w^{\prime}=\nabla V(w)$. Since $w_{n}(0)=\frac{z_{n}(0)}{\left\|z_{n}\right\|_{\infty}} \in l_{S}^{1}$, we have $w(t)=C \varphi_{V}\left(t+\tau_{0, V}\right)$, for a suitable $C>0$. Writing $z_{n}(t)=r_{n}(t) \varphi_{V}(t+$ $\left.\omega_{n}(t)\right)$, with $\omega_{n}(0)=\tau_{0, V}$, since $2 V\left(\varphi_{V}\right) \equiv 1$ we have

$$
\int_{0}^{T} \frac{\left\langle R\left(t, z_{n}(t)\right) \mid z_{n}(t)\right\rangle}{2 V\left(z_{n}(t)\right)} d t=\int_{0}^{T} \frac{\left.\left.\left\langle R\left(t, r_{n}(t) \varphi_{V}\left(t+\omega_{n}(t)\right)\right)\right| \varphi_{V}\left(t+\omega_{n}(t)\right)\right)\right\rangle}{r_{n}(t)} d t .
$$

Since $w_{n} \rightarrow w$ uniformly, on one hand we have that $\frac{r_{n}}{\left\|z_{n}\right\|_{\infty}} \rightarrow C$ uniformly; on the other hand, $\omega_{n}$ converges to a constant, so that $\omega_{n} \rightarrow \tau_{0, V}$ uniformly (recall that $\left.\omega_{n}(0)=\tau_{0, V}\right)$. By Fatou's lemma, we thus obtain, in view of the properties of the inferior limit,

$$
\begin{aligned}
0 & \geq \liminf _{n \rightarrow+\infty}\left\|z_{n}\right\|_{\infty} \mathcal{R}_{n} \\
& \geq \int_{0}^{T} \liminf _{n \rightarrow+\infty} \frac{\left.\left.\left\langle R\left(t, r_{n}(t) \varphi_{V}\left(t+\omega_{n}(t)\right)\right)\right| \varphi_{V}\left(t+\omega_{n}(t)\right)\right)\right\rangle}{\frac{r_{n}(t)}{\left\|z_{n}\right\|_{\infty}}} d t \\
& \geq \int_{0}^{T} \liminf _{(\lambda, \omega) \rightarrow\left(+\infty, \tau_{0, V}\right)}\left\langle R\left(t, \lambda \varphi_{V}(t+\omega)\right) \mid \varphi_{V}(t+\omega)\right\rangle d t,
\end{aligned}
$$

contradicting $\mathcal{J}^{-}\left(\tau_{0, V}\right)>0$.

We conclude this section with some observations about possible extensions and variants of our main result.

Remark 2.5. We first notice that, to perform the shooting technique, it would be sufficient to assume, instead of (16), an $L^{1}$-sublinearity condition of the following type: for every $\epsilon>0$, there exists $\eta_{\epsilon} \in L^{1}(0, T)$ such that, for almost every $t \in[0, T]$ and every $z \in \mathbb{R}^{2}$,

$$
|R(t, z)| \leq \epsilon|z|+\eta_{\epsilon}(t) .
$$

However, in order for the integral in $\mathcal{J}^{+}$(resp. $\mathcal{J}^{-}$) to make sense, a suitable $L^{1}$-control from above (resp. below) on $R(t, \cdot)$ is in this case needed (see, for instance, [3]). For the sake of simplicity, we have preferred to assume the twosided boundedness condition (16).

Remark 2.6. In principle, we can deal with more general (nonlinear, homogeneous) boundary conditions on cones, as well. For instance, we can replace each of the lines $l_{S}$ and $l_{A}$ with the union of two half-lines emanating from the origin. The features of the resonance phenomenon for this "polygonal" problem have been extensively discussed in [11, Section 4], a crucial role being played by the mutual position of the half-lines. The Landesman-Lazer conditions can be formulated with the same spirit as in Theorems 2.1 and 2.3 (see also Figure 6 at the end of the paper). 
Remark 2.7. The central point of our proof is given by Lemma 2.4, which provides a sharp interpretation of the Landesman-Lazer condition in terms of the (noninteger) number of windings of large solutions to (21) around the origin. This property was already highlighted (for complete windings) in [3, Section 4], and then applied, together with the Poincaré-Birkhoff fixed point theorem, to obtain multiplicity of $T$-periodic solutions for planar Hamiltonian systems like $J z^{\prime}=\nabla_{z} H(t, z)$, with $\nabla_{z} H(t, 0) \equiv 0$ and exhibiting a "gap" between zero and infinity. In the same spirit, we can use our Landesman-Lazer conditions to obtain multiple solutions for resonant Sturm-Liouville problems, improving the results in [11, Section 5]. Notice that, in this case, the shooting technique used does not require any Hamiltonian structure.

\section{Corollaries for scalar second order ODEs}

In this section, we explicitly state some corollaries of Theorems 2.1 and 2.3 for resonant problems associated with the scalar second order asymmetric equation

$$
u^{\prime \prime}+\mu u^{+}-\nu u^{-}+r(t, u)=0,
$$

with $\mu, \nu$ positive $e^{1}$ constants, $u^{ \pm}=\max \{ \pm u, 0\}$ and $r(t, u)$ a Carathéodory function which is $L^{1}$-bounded in the $u$-variable, that is, for some $\xi \in L^{1}(0, T)$,

$$
|r(t, u)| \leq \xi(t)
$$

for almost every $t \in[0, T]$ and every $u \in \mathbb{R}$. In this way, equation (23) enters the planar setting used in Section 2, with the positions $z=(u, v), V(z)=\frac{1}{2}\left[v^{2}+\right.$ $\left.\mu\left(u^{+}\right)^{2}+\nu\left(u^{-}\right)^{2}\right]$ and $R(t, z)=(r(t, u), 0)$. It is thus possible to use Theorems 2.1 and 2.3 to give existence results at resonance when (23) is considered jointly with general Sturm-Liouville boundary conditions of the type

$$
u(0) \cos \alpha-u^{\prime}(0) \sin \alpha=0, \quad u(T) \cos \beta-u^{\prime}(T) \sin \beta=0,
$$

for $\alpha, \beta \in[0,2 \pi[$. We choose to focus our attention on three particular situations: the Dirichlet BVP $(\alpha=\beta=0)$, the Neumann one $\left(\alpha=\beta=\frac{\pi}{2}\right)$ and a mixed boundary value problem of Robin type $\left(\alpha=0, \beta=\frac{\pi}{2}\right)$.

\footnotetext{
${ }^{1}$ With a little abuse in the notation, in the following we will speak about Dancer-Fučik spectrum of a boundary value problem associated with (23) restricting our attention to the open first quadrant $\left(\mathbb{R}^{+}\right)^{2}$ of the $(\mu, \nu)$-plane (see formulas $\left.(29),(33),(40)\right)$. For some SturmLiouville boundary conditions, resonance could also appear when $\mu \leq 0$ or $\nu \leq 0$ (for instance, this is the case for the Dirichlet problem associated with $\left.u^{\prime \prime}+\left(\frac{\pi}{T}\right)^{2} u^{+}=0\right)$. Landesman-Lazer type results could be established in these situations as well, on the lines of $[2,14]$.
} 
For our analysis, we fix a reference solution $\phi_{\mu, \nu}(t)$ to the homogeneous equation $u^{\prime \prime}+\mu u^{+}-\nu u^{-}=0$ by setting

$$
\phi_{\mu, \nu}(t)= \begin{cases}\frac{1}{\sqrt{\mu}} \sin (\sqrt{\mu} t) & \text { if } \quad t \in\left[0, \frac{\pi}{\sqrt{\mu}}\right] \\ \frac{1}{\sqrt{\nu}} \sin \left(\sqrt{\nu}\left(\frac{\pi}{\sqrt{\mu}}-t\right)\right) & \text { if } \quad t \in\left[\frac{\pi}{\sqrt{\mu}}, \frac{\pi}{\sqrt{\mu}}+\frac{\pi}{\sqrt{\nu}}\right]\end{cases}
$$

(extending the definition by $\left(\frac{\pi}{\sqrt{\mu}}+\frac{\pi}{\sqrt{\nu}}\right)$-periodicity); notice that all the other solutions are of the form $u(t)=C \phi_{\mu, \nu}(t+\theta)$, for $C \geq 0$ and $\theta \in \mathbb{R}$. Of course, recalling the notation in Section 2 , we have $\varphi_{V}(t)=\left(\phi_{\mu, \nu}(t), \phi_{\mu, \nu}^{\prime}(t)\right)$.

Lastly, we set, for an $L^{\infty}$-function $\zeta(t)$,

$$
\mathcal{A}_{\zeta}^{-}=\int_{\{\zeta>0\}}\left(\liminf _{x \rightarrow+\infty} r(t, x)\right) \zeta(t) d t+\int_{\{\zeta<0\}}\left(\limsup _{x \rightarrow-\infty} r(t, x)\right) \zeta(t) d t
$$

and

$$
\mathcal{A}_{\zeta}^{+}=\int_{\{\zeta>0\}}\left(\limsup _{x \rightarrow+\infty} r(t, x)\right) \zeta(t) d t+\int_{\{\zeta<0\}}\left(\liminf _{x \rightarrow-\infty} r(t, x)\right) \zeta(t) d t,
$$

where $\{\zeta>0\}$ (resp. $\{\zeta<0\})$ stands for the subset of $[0, T]$ where $\zeta(t)$ is positive (resp. negative). Notice that, similarly as for $\mathcal{J}^{ \pm}$, we have $\mathcal{A}_{\zeta}^{-} \leq \mathcal{A}_{\zeta}^{+}$.

3.1. The Dirichlet problem. In this subsection, we consider equation (23) together with Dirichlet boundary conditions

$$
u(0)=u(T)=0 .
$$

According to the notation used in Section 2, we thus have $l_{S}=l_{A}=\{0\} \times \mathbb{R}$ and

$$
\tau_{0, V}=0, \quad \tau_{1, V}=\frac{\pi}{\sqrt{\mu}}, \quad \tau_{2, V}=\frac{\pi}{\sqrt{\nu}}, \quad \sigma_{1, V}=\sigma_{2, V}=0 .
$$

Hence, the resonance assumptions (9), (11) and (10) (this last coinciding with (12)) translate, respectively, into the following:

$$
\begin{gathered}
(k+1) \frac{\pi}{\sqrt{\mu}}+k \frac{\pi}{\sqrt{\nu}}=T, \\
k \frac{\pi}{\sqrt{\mu}}+(k+1) \frac{\pi}{\sqrt{\nu}}=T, \\
(k+1)\left(\frac{\pi}{\sqrt{\mu}}+\frac{\pi}{\sqrt{\nu}}\right)=T,
\end{gathered}
$$


for a nonnegative integer $k$. Henceforth, we use the notation

$$
\Sigma_{D}=\left\{(\mu, \nu) \in\left(\mathbb{R}^{+}\right)^{2} \mid(26),(27) \text { or (28) hold for some integer } k \geq 0\right\},
$$

namely $\Sigma_{D}$ is the Dancer-Fučik spectrum associated with problem (23)-(25). The possible eigenfunctions corresponding to (26)-(28) are given by the positive multiples of

$$
\phi_{D}(t)=\phi_{\mu, \nu}(t), \quad \psi_{D}(t)=\phi_{\mu, \nu}\left(t+\frac{\pi}{\sqrt{\mu}}\right) .
$$

Precisely, $\phi_{D}$ and $\psi_{D}$ are, respectively, the eigenfunctions corresponding to conditions (26) and (27), while they are both eigenfunctions if (28) holds.

In this setting, the results stated in Section 2 can be rephrased into the following statement.

Theorem 3.1. Assume that $(\mu, \nu) \in \Sigma_{D}$ and let $r(t, u)$ satisfy (24). Then, sufficient conditions for the solvability of problem (23)-(25) are the following:

- if (26) holds,

$$
\begin{array}{lll}
\mu>\nu & \text { and } & \mathcal{A}_{\phi_{D}}^{+}<0 \\
\mu<\nu & \text { and } & \mathcal{A}_{\phi_{D}}^{-}>0
\end{array}
$$

- if (27) holds,

$$
\begin{array}{lll}
\mu>\nu & \text { and } & \mathcal{A}_{\psi_{D}}^{-}>0 \\
\mu<\nu & \text { and } & \mathcal{A}_{\psi_{D}}^{+}<0
\end{array}
$$

- if either (28) holds or $\mu=\nu$,

$$
\mathcal{A}_{\phi_{D}}^{-}>0, \quad \mathcal{A}_{\psi_{D}}^{-}>0, \quad \text { or } \quad \mathcal{A}_{\phi_{D}}^{+}<0, \quad \mathcal{A}_{\psi_{D}}^{+}<0 .
$$

Proof. Using standard properties of the inferior and superior limits (see also [10, Proposition 3.1]), one can see that the conditions on $\mathcal{J}^{ \pm}$can be translated into the given assumptions on $\mathcal{A}^{ \pm}$.

We observe that, when $\mu=\nu$, one has $\phi_{D}=-\psi_{D}$, so that, defining, for an $L^{\infty}$-function $\zeta(t)$

$$
\mathcal{B}_{\zeta}^{-}=\int_{\{\zeta>0\}}\left(\liminf _{x \rightarrow-\infty} r(t, x)\right) \zeta(t) d t+\int_{\{\zeta<0\}}\left(\limsup _{x \rightarrow+\infty} r(t, x)\right) \zeta(t) d t
$$

and

$$
\mathcal{B}_{\zeta}^{+}=\int_{\{\zeta>0\}}\left(\limsup _{x \rightarrow-\infty} r(t, x)\right) \zeta(t) d t+\int_{\{\zeta<0\}}\left(\liminf _{x \rightarrow+\infty} r(t, x)\right) \zeta(t) d t
$$


it is possible to write condition (30) as

$$
\mathcal{B}_{\phi_{D}}^{+}<0<\mathcal{A}_{\phi_{D}}^{-} \quad \text { or } \quad \mathcal{A}_{\phi_{D}}^{+}<0<\mathcal{B}_{\phi_{D}}^{-}
$$

or, equivalently,

$$
\mathcal{B}_{\psi_{D}}^{+}<0<\mathcal{A}_{\psi_{D}}^{-} \quad \text { or } \quad \mathcal{A}_{\psi_{D}}^{+}<0<\mathcal{B}_{\psi_{D}}^{-}
$$

forms which are reminiscent of the original result by Landesman and Lazer [16]. We provide a pictorial description of Theorem 3.1 in Figure 3 below, referring to Remark 3.4 for a detailed explanation.

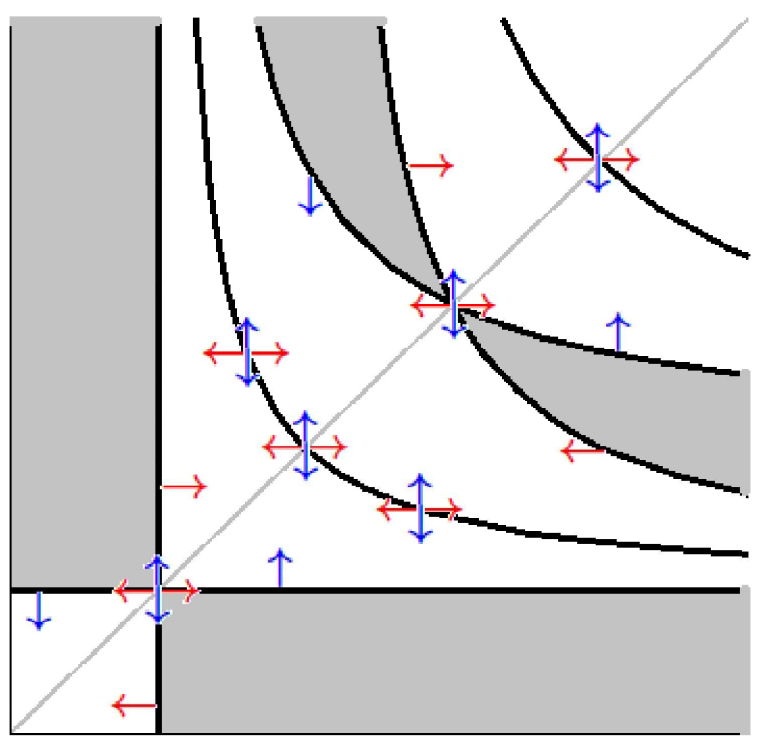

Figure 3: The first curves of the Dancer-Fučik spectrum for Dirichlet boundary conditions are depicted in the first quadrant of the $(\mu, \nu)$-plane; the arrows illustrate the role of the Landesman-Lazer conditions (see Remark 3.4). The diagonal is drawn to highlight the symmetries of the spectrum.

3.2. The Neumann problem. We now turn to consider (23) together with Neumann boundary conditions

$$
u^{\prime}(0)=u^{\prime}(T)=0 .
$$

Here, we have $l_{S}=l_{A}=\mathbb{R} \times\{0\}$ and

$$
\tau_{0, V}=\frac{\pi}{2 \sqrt{\mu}}, \quad \tau_{1, V}=\tau_{2, V}=\frac{1}{2}\left(\frac{\pi}{\sqrt{\mu}}+\frac{\pi}{\sqrt{\nu}}\right), \quad \sigma_{1, V}=\sigma_{2, V}=0 .
$$


Accordingly, the resonance assumptions (9)-(12) translate into the unique one

$$
\frac{k+1}{2}\left(\frac{\pi}{\sqrt{\mu}}+\frac{\pi}{\sqrt{\nu}}\right)=T,
$$

for a nonnegative integer $k$. In this case, the Dancer-Fučik spectrum is thus given by

$$
\Sigma_{N}=\left\{(\mu, \nu) \in\left(\mathbb{R}^{+}\right)^{2} \mid(32) \text { holds for some integer } k \geq 0\right\},
$$

with corresponding eigenfunctions

$$
\phi_{N}(t)=\phi_{\mu, \nu}\left(t+\frac{\pi}{2 \sqrt{\mu}}\right), \quad \psi_{N}(t)=\phi_{\mu, \nu}\left(t+\frac{\pi}{\sqrt{\mu}}+\frac{\pi}{2 \sqrt{\nu}}\right) .
$$

In this setting, the results of Section 2 translate into the following statement.

Theorem 3.2. Assume that $(\mu, \nu) \in \Sigma_{N}$ and let $r(t, u)$ satisfy (24). Then, problem (23)-(31) has a solution if the following condition is satisfied:

$$
\mathcal{A}_{\phi_{N}}^{-}>0, \quad \mathcal{A}_{\psi_{N}}^{-}>0, \quad \text { or } \quad \mathcal{A}_{\phi_{N}}^{+}<0, \quad \mathcal{A}_{\psi_{N}}^{+}<0 .
$$

Incidentally, we notice that for $\mu=\nu$ one has $\phi_{N}=-\psi_{N}$, so that it is possible to rewrite condition (34) using the definitions of $\mathcal{B}^{ \pm}$previously introduced. As for the Dirichlet problem, we give a visual representation of the statement in Figure 4 below (see also Remark 3.4).

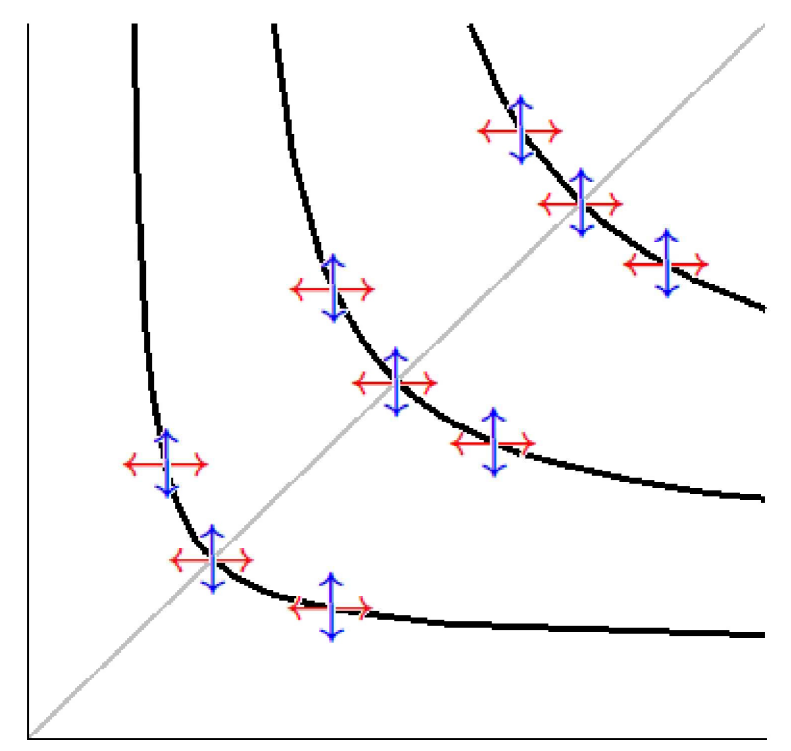

Figure 4: The situation for the Neumann boundary value problem. 
3.3. A mixed problem. As a final example, we now consider (23) together with mixed boundary conditions of Robin type

$$
u(0)=u^{\prime}(T)=0 .
$$

Here, we have $l_{S}=\{0\} \times \mathbb{R}, l_{A}=\mathbb{R} \times\{0\}$ and

$$
\tau_{0, V}=0, \quad \tau_{1, V}=\sigma_{1, V}=\frac{\pi}{2 \sqrt{\mu}}, \quad \tau_{2, V}=\sigma_{2, V}=\frac{\pi}{2 \sqrt{\nu}} .
$$

Accordingly, the resonance assumptions (9), (10), (11) and (12) translate, respectively, into

$$
\begin{array}{r}
\frac{2 k+1}{2} \frac{\pi}{\sqrt{\mu}}+k \frac{\pi}{\sqrt{\nu}}=T, \\
(k+1) \frac{\pi}{\sqrt{\mu}}+\frac{2 k+1}{2} \frac{\pi}{\sqrt{\nu}}=T, \\
k \frac{\pi}{\sqrt{\mu}}+\frac{2 k+1}{2} \frac{\pi}{\sqrt{\nu}}=T, \\
\frac{2 k+1}{2} \frac{\pi}{\sqrt{\mu}}+(k+1) \frac{\pi}{\sqrt{\nu}}=T,
\end{array}
$$

for a nonnegative integer $k$. Accordingly, one can analogously define a DancerFučik spectrum

$$
\Sigma_{M}=\left\{(\mu, \nu) \in\left(\mathbb{R}^{+}\right)^{2} \mid(36),(37),(38) \text { or (39) hold for some } k \geq 0\right\}
$$

and the corresponding eigenfunctions are $\phi_{M}(t)=\phi_{\mu, \nu}(t), \psi_{M}(t)=\phi_{\mu, \nu}\left(t+\frac{\pi}{\sqrt{\mu}}\right)$. In this setting, the results of Section 2 translate into the following statement.

Theorem 3.3. Assume that $(\mu, \nu) \in \Sigma_{M}$ and let $r(t, u)$ satisfy (24). Then, sufficient conditions for the solvability of problem (23)-(35) are the following:

- if (36) or (37) holds,

$$
\begin{array}{lll}
\mu>\nu & \text { and } & \mathcal{A}_{\phi_{M}}^{+}<0 \\
\mu<\nu & \text { and } & \mathcal{A}_{\phi_{M}}^{-}>0
\end{array}
$$

- if (38) or (39) holds,

$$
\begin{array}{lll}
\mu>\nu & \text { and } & \mathcal{A}_{\psi_{M}}^{-}>0 \\
\mu<\nu & \text { and } & \mathcal{A}_{\psi_{M}}^{+}<0
\end{array}
$$

- if $\mu=\nu$, independently of the resonance condition assumed,

$$
\mathcal{A}_{\phi_{M}}^{-}>0, \quad \mathcal{A}_{\psi_{M}}^{-}>0, \quad \text { or } \quad \mathcal{A}_{\phi_{M}}^{+}<0, \quad \mathcal{A}_{\psi_{M}}^{+}<0 .
$$


Notice that, since when $\mu=\nu$ one has $\phi_{M}=-\psi_{M}$, again one can rewrite condition (41) in the equivalent form involving $\mathcal{B}^{ \pm}$. Figure 5 below illustrates the statement; once again, we refer the reader to Remark 3.4.

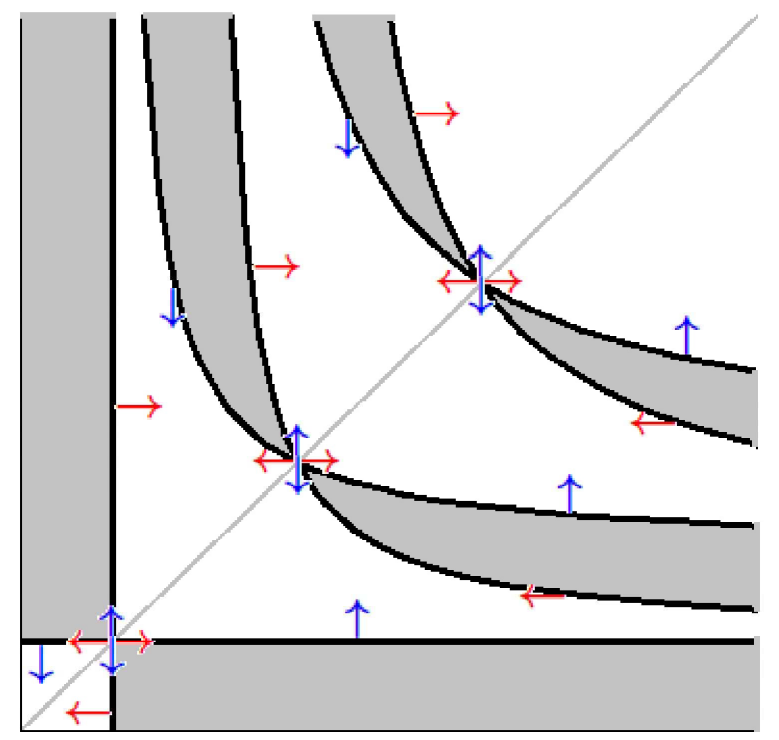

Figure 5: The situation for the boundary conditions (35).

We conclude the paper with a detailed explanation of the perspective suggested by the figures in this section.

Remark 3.4. In Figures 3, 4 and 5, the first curves of the corresponding Dancer-Fučik spectra are represented in the first quadrant of the $(\mu, \nu)$-plane. On such curves, the autonomous problem $u^{\prime \prime}+\mu u^{+}-\nu u^{-}=0$ (with prescribed boundary conditions (25), (31) or (35)) has a nontrivial solution and the corresponding perturbed problem $u^{\prime \prime}+\mu u^{+}-\nu u^{-}+r(t, u)=0$ may not be solvable. On the other hand, some regions out of the spectrum are shaded, corresponding to couples $(\mu, \nu)$ such that, though the autonomous problem admits only the trivial solution, the solvability of the perturbed one is still not guaranteed in general (in the following, we will refer to them as "special resonance regions"). This is a well-known phenomenon $[4,13]$ (see also [11]), whose appearance depends on the given boundary conditions. In fact, already at first sight, our figures display different features:

- in Figure 5, namely for mixed boundary conditions $u(0)=u^{\prime}(T)=0$, curves appear in couples intersecting on the diagonal, each of them delimiting a special resonance region (this is indeed the typical situation for a general Sturm-Liouville problem); 
- in Figure 3, namely for Dirichlet boundary conditions, we see a pattern alternating couples of curves, again delimiting special resonance regions, and single curves;

- in Figure 4, namely for Neumann boundary conditions, only single curves occur and no special resonance regions are present (incidentally, this is the same situation as for periodic boundary conditions).

We can intuitively think of a single curve as the "limit case" when two distinct curves coincide and there is no room in between for special resonance regions. With this in mind, we can interpret the Landesman-Lazer conditions as a way to make the nonlinearity "escape" from the spectrum - as usual - and from the special resonance regions (when present), as the arrows in the figures suggest. To be precise, the correspondence between Landesman-Lazer conditions and arrows is as follows:

- $\mathcal{A}_{\phi}^{-}>0$ (resp., $\left.\mathcal{A}_{\phi}^{+}<0\right)$ : red arrow to the right (resp., to the left);

- $\mathcal{A}_{\psi}^{-}>0$ (resp., $\mathcal{A}_{\psi}^{+}<0$ ): blue arrow to the top (resp., to the bottom).

Focusing on one special resonance region, we notice that the eigenfunction involved in the Landesman-Lazer condition is always the same (and is the one naturally associated) on each curve delimiting it. However, the sign needed in the inequality changes: this is coherent with the fact that, in order to escape from the special resonance region, one has to move in opposite ways according to whether $\mu<\nu$ or $\mu>\nu$. This pictorial interpretation also makes clear what is going on when two distinct curves "degenerate" into a single one or when $\mu=\nu$. Precisely: on one hand two Landesman-Lazer conditions are needed (since the two curves are intersecting); on the other hand, there is more room to escape from the (possibly empty) special resonance region, so that both the sign for the inequalities are admissible.

We finally mention that this approach can be a guideline when dealing with nonlinear boundary conditions giving rise to more complicated pictures for the associated spectrum. For instance (compare with Remark 2.6) we can consider equation (23) together with the boundary condition

$$
u^{\prime}(0)=0, u(0) \geq 0 \quad \text { or } \quad u(0)=0, u^{\prime}(0) \geq 0
$$

and

$$
u^{\prime}(T)=0, u(T) \leq 0 \quad \text { or } \quad u(T)=0, u^{\prime}(T) \leq 0
$$

(namely, $u(t)$ starts from one positive semi-axis and arrives on one negative one), whose corresponding spectrum has been depicted in [11, Figure 9]. The Landesman-Lazer conditions to be imposed are easily derived just by "adding arrows" according to the previous philosophy (see Figure 6 below for some hints). For the sake of briefness, we leave the details to the interested reader. 


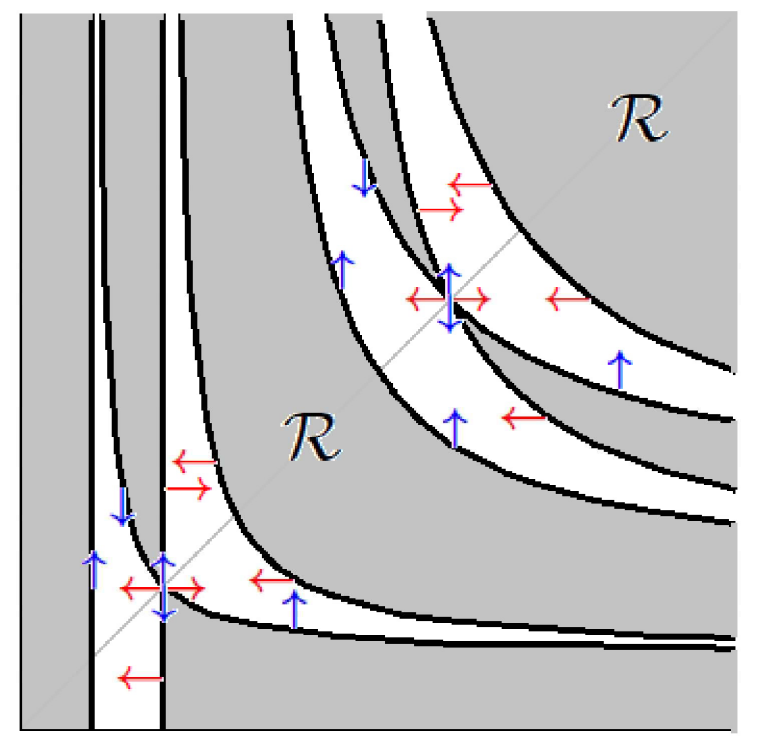

Figure 6: The polygonal problem corresponding to (42) and (43). Compared with the spectrum of the Dirichlet problem in Figure 3, new special resonance regions (denoted by $\mathcal{R}$ ) appear, delimited by couples of asymmetric curves. Intuitively, such curves may be interpreted as coming from the splitting of the single ones in the Dirichlet case. Accordingly, one-sided Landesman-Lazer conditions (with respect to one eigenfunction) are now needed on each of these curves. On the other hand, we recognize the usual picture on couples of symmetric curves, intersecting on the diagonal.

\section{References}

[1] Asakawa, H., Landesman-Lazer type problems for Fučík's spectrum. Nonlinear Anal. 26 (1996), $407-414$.

[2] Boscaggin, A., One-signed harmonic solutions and sign-changing subharmonic solutions to scalar second order differential equations. Adv. Nonlinear Stud. 12 (2012), $445-463$.

[3] Boscaggin, A. and Garrione, M., Resonance and rotation numbers for planar Hamiltonian systems: multiplicity results via the Poincaré-Birkhoff theorem. Nonlinear Anal. 74 (2011), 4166 - 4185.

[4] Dancer, E. N., Boundary-value problems for weakly nonlinear ordinary differential equations. Bull. Austral. Math. Soc. 15 (1976), 321 - 328.

[5] Dong, Y., Landesman-Lazer conditions for Sturm-Liouville BVPs with generalized Fucik types of resonant points of asymptotically positively homogeneous equations. Z. Angew. Math. Phys. 53 (2002), $692-703$. 
[6] Drábek, P., Landesman-Lazer condition for nonlinear problems with jumping nonlinearities. J. Diff. Equ. 85 (1990), 186 - 199.

[7] Fabry, C., Landesman-Lazer conditions for periodic boundary value problems with asymmetric nonlinearities. J. Diff. Equ. 116 (1995), 405 - 418.

[8] Fabry, C. and Fonda, A., Periodic solutions of perturbed isochronous Hamiltonian systems at resonance. J. Diff. Equ. 214 (2005), 299 - 325.

[9] Fonda, A., Positively homogeneous Hamiltonian systems in the plane. J. Diff. Equ. 200 (2004), 162 - 184.

[10] Fonda, A. and Garrione, M., Double resonance with Landesman-Lazer conditions for planar systems of ordinary differential equations. J. Diff. Equ. 250 (2011), $1052-1082$.

[11] Fonda, A. and Garrione, M., Generalized Sturm-Liouville boundary conditions for first order differential systems in the plane. Topol. Methods Nonlinear Anal. 42 (2013), $293-325$.

[12] Fonda, A. and Mawhin, J., Planar differential systems at resonance. Adv. Diff. Equ. 11 (2006), 1111 - 1133.

[13] Fučik, S., Boundary value problems with jumping nonlinearities. Časopis Pěst. Mat. 101 (1976), 69 - 87.

[14] Garrione, M., Resonance at the first eigenvalue for first-order systems in the plane: vanishing Hamiltonians and the Landesman-Lazer condition. Diff. Integral Equ. 25 (2012), 505 - 526.

[15] Genoud, F. and Rynne, B., Landesman-Lazer conditions at half-eigenvalues of the p-Laplacian. J. Diff. Equ. 254 (2013), $3461-3475$.

[16] Landesman, E. and Lazer, A. C., Nonlinear perturbations of linear elliptic boundary value problems at resonance. J. Math. Mech. 19 (1970), 609 - 623.

[17] Rynne, B., Non-resonance conditions for semilinear Sturm-Liouville problems with jumping non-linearities. J. Diff. Equ. 170 (2001), 215 - 227.

Received August 29, 2014 